\title{
Reflections on the Pharmacotherapy Aspects of Cov-2 Variants
}

\section{Seghatchia J*}

International Consultancy in Innovative Manufacturing and Quality/ Safety of Blood-Derived Bioproducts, UK

*Corresponding author: J erard Seghatchian, International Consultancy in Innovative Manufacturing and Quality/ Safety of Blood-Derived Bioproducts. London, England, UK

Received: February 08, 2021; Accepted: February 24, 2021; Published: March 03, 2021

\section{Short Commentary}

The coronavirus SARS-CoV-2 epitomises the perfect example of a storm, a nightmare pandemic scenario that requires rescue plans for the infected populations. This is of particular relevance as newer fast spreading variants appearing with almost 10 times faster, despite numerous precautionary measures and restriction being in place to reduce this happen.

An armamentarium of anti-viral, anti-inflammatory, and antithrombotic drugs are being recruited, through artificial intelligence tools in big data, patterns and procedures to survive the problem; research in plasmapheresis-based exchange technologies to decrease inflammatory mediators; in-line affinity column absorption to prepare neutralizing antibody concentrate against the variants strains of this virus, as the most useful direct intervention and more recently novel developments in vaccines with reported efficacies in the range $62 \%$ to $95 \%$, ie. to simian adenovirus-vectored and the mRNA vaccines all with partial success with the implementation of personal protective equipment and physical distancing.

A preliminary analysis of published data on the relationship between $\mathrm{T}$ cell lymphopenia and the outcomes of coronavirus infections, that have been stratified in various ways to measure cumulative $\left[\mathrm{CD} 4{ }^{+} \mathrm{CD} 8\right]$ counts: (1) mild to moderate v. severe disease; (2) survivors v. non-survivors; (3) non-severe v. severe cases; and (4) infected patients v. healthy controls suggest:

Firstly, the severity of T-cell depletion correlates with a worse patient outcome for SARS. Secondly, the degree of lymphopenia appears to associate with a cytokine driven hyperinflammatory cascade triggered by coronaviruses. Thirdly, quantitative T-cell lymphodepletion may be a surrogate marker of hyper- inflammation and has a potential role to identify the timing of when resource intensive clinical treatments should be applied and be effective. Fourthly, recognition of elevated regulatory T-cells (Tregs) as an important T-cell subset to modulate immune responses and may lower the risk of respiratory viral infections in the elderly.

Moreover, evidence is accumulating that the onset of SARS becomes highly critical in a smaller percentage of patients (estimated $1.4 \%$ infection mortality rate) who are susceptible innately to an intense cytokine/chemokine inflammatory response; and with multiple risk factors. The notion thought to be driven by the virus in the lungs causing massive pulmonary edema and requiring mechanical support by ventilator or even Extracorporeal Membrane Oxygenation (ECMO). Given the large number of infections in the population encompassed by SARS CoV-2, this percentage of patients with intense inflammation although small translates to a major stressor on the limited availability of hospital medical ICU beds and intensivists. Therapeutically, a miscellany of monoclonal antibodies, recombinant protein and antiviral drugs are being tried to attenuate the adverse effects of the hyperinflammatory cascade. These include both tocilizumab, a monoclonal antibody and inhibitor of the interleukin- 6 receptor and the recombinant protein anakinra, an interleukin-1 receptor antagonist of the cytokine inflammatory cascade. Remdesivir is an anti-viral prodrug, an adenosine nucleotide inhibitor of the SARS-CoV-2 RNA dependent RNA Polymerase (RdRp) essential for viral replication. The drug has reported modest therapeutic effects, shortening the average duration of hospitalisation by four days. Moreover, to attenuate the host inflammatory factors of SARS, future R\&D are already progressing well with specialised immunosorbent devices incorporated into extracorporeal circuits for hemopurification. One such product of potential utility is a biocompatible porous polymer adsorbent micro bead. Undoubtedly, as is in practice with such an advanced technologies established for many decades the manufacturers do take the responsibly to ensure such hemopurification devices in development are secured in the most effective standardised way under strict GMP regulatory adherence.

Another matter of pharmacological concern is the use of painkillers to dampen the side effect of vaccination. For most people, the side effects of the vaccine are mild or moderate and last only a day or two, though in a limited cases in some people the side effects might them feel like they have the flu, affecting their ability to perform daily activities; These side effects are the signs of an appropriate recognition and immune reactions to the active principle of vaccine, is teaching the recipient immune system how to recognize and attack SARSCoV-2, the coronavirus that causes COVID-19, when encountered. In the context of the use of painkiller when faced with a couple of days of flu-like discomfort, such as using ibuprofen (Advil, Motrin) or acetaminophen (Tylenol) to relieve their fever and pain to reduce the side effects of the vaccine, is not warranted as there a chance that they'll also depress the immune system's beneficial response to vaccination. Moreover using a pain reliever before the vaccine injection to head off the discomfort that the drugs may dampen the inflammatory response, fever and aches with reduced inflammation, unwarranted as it may also be a lower immune response to the vaccine. Moreover, Non-Steroidal Anti-Inflammatory Drugs (NSAIDs), which include ibuprofen, reduced the production of antibodies and other aspects of the immune response to SARS-CoV-2. 
Basically antibodies are proteins made by the immune system to fight viruses like CoV-2. The COVID-19 vaccines stimulate the body to produce antibodies that specifically target the coronavirus without causing disease. While some fever-reducing pain medications may blunt the antibody response to vaccination, but it's not clear what this means in the real world. While more research is needed but based on the existing data on the COVID-19 vaccine studies that have already been done it is clear that taking a pain reliever after injection, if needed, may not cause that much of a problem. To conclude in short as of 10 January 2021, over 1,941,757 people have died so far from the coronavirus COVID-19 outbreak, all from a miniscule RNA virus $0.125 \mathrm{um}$ in diameter. The virus has truly challenged mankind, which despite our ingenuity, the infections and deaths are continuing to rise exponentially in the colder months, facilitated by cohabitation and travel. Despite these rising numbers, we should be sanguine about the prospects for a better future. The Calvary has arrived with the ongoing mass distribution of multiple vaccines and warmer weather only several months away. With encouragement, we should be cautiously optimistic about the longer-term actions of the public for responsibly enhancing the use of personal protective equipment, physical distancing and hygienic practices. Add on the targeted use of pharmaceuticals and plasmapheresis technologies to mitigate infections and prevent deaths. SARS-CoV-2 is only an awakening with numerous forewarnings, infection disease, Yet not to underestimate our resourcefulness. With advancing developmental technologies, we should improve in the future. 